\title{
Ensino online em tempos de pandemia: a opinião de universitários quanto aos
}

\section{desafios encontrados}

Online education in times of pandemic: the opinion of college students related to the challenges

founds

Docencia online en tiempos of pandemia: la opinión de los universitários con los desafios

encontrados

Recebido: 26/07/2021 | Revisado: 31/07/2021 | Aceito: 02/08/2021 | Publicado: 07/08/2021

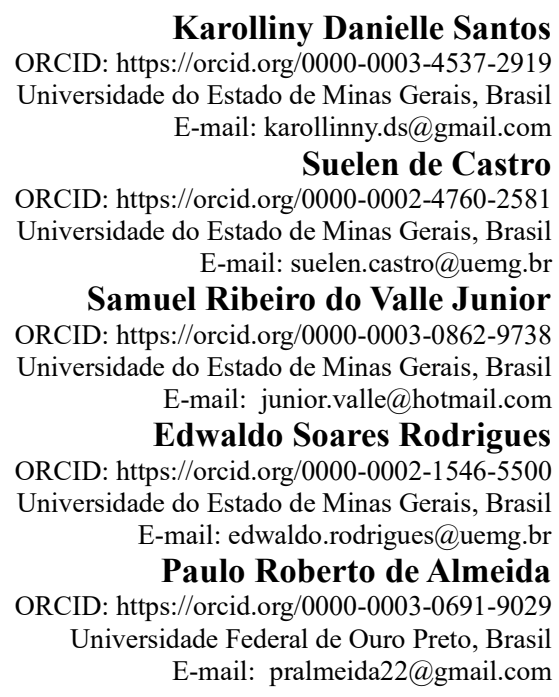

\section{Resumo}

Devido a pandemia do COVID-19 e a necessidade de isolamento social, o ensino presencial foi substituído pelo ensino online nas universidades. Diante disso, o presente artigo tem por objetivos conhecer o ambiente que os alunos estão inseridos durante as aulas remotas, assim como analisar a opinião desses universitários com relação aos impactos da pandemia na sua rotina de aprendizagem. Para isso, foi aplicado um questionário online por meio da plataforma Microsoft Forms aos estudantes regularmente matriculados no curso de Engenharia da Computação da Universidade do Estado de Minas Gerais - UEMG. Os resultados mostraram que as maiores dificuldades encontradas pelos discentes durante as aulas online foram a de adaptação as aulas remotas, bem como fatores psicológicos. Apesar da internet ser uma fragilidade durante o ensino remoto, pelas respostas dos estudantes fatores emocionais mostraramse mais relevantes nesse período de pandemia. A análise dos dados obtidos com os questionários aplicados mostrou que embora plataformas adequadas para o ensino online, assim como uma boa conexão com a internet sejam imprescindíveis para que as aulas remotas aconteçam fatores emocionais decorrentes do isolamento social, adaptação as aulas e o ambiente de estudo foram os que mais atrapalharam o processo de aprendizagem dos alunos durante o período de pandemia.

Palavras-chave: Pandemia; COVID; Ensino online; Aprendizagem online; Ensino.

\begin{abstract}
Due to the COVID-19 pandemic and the need for social isolation, face-to-face teaching was replaced by online education at universities. Thus, this article aims to know the environment in which students are inserted during remote classes and analyze the opinion of these academics about the effects of the pandemic on their daily learning. Therefore, an online questionnaire through Microsoft Forms was applied to students regularly enrolled in the Computer Engineering course at UEMG. The results show that the most difficulties found by students during online classes were adaptation to remote classes, and psychological factors. Although the internet is weakness during teaching, according to the response of these students, emotional factors were more relevant in this period. The analysis of the data obtained from the questionnaires applied showed that although adequate platforms for online teaching, as well as a good internet connection, are essential for remote classes to take place, emotional factors arising
\end{abstract}


from social isolation, adaptation to classes and the study environment were those that most disturbed learning process of the students during the pandemic time.

Keywords: Pandemic; COVID-19; Online education; Online learning; Teaching.

\section{Resumen}

Debido a la pandemia de COVID-19 y la necesidad de aislamiento social, la enseñanza presencial fue reemplazada por la educación en línea en las universidades. Así, este artículo tiene como objetivo conocer el entorno en el que se insertan los estudiantes durante las clases a distancia y analizar la opinión de estos académicos sobre los efectos de la pandemia en su aprendizaje diario. Por ello, se aplicó un cuestionario online a través de Microsoft Forms a los alumnos matriculados habitualmente en el curso de Ingeniería Informática de la UEMG. Los resultados muestran que las mayores dificultades encontradas por los estudiantes durante las clases a distancia fueron la adaptación a las clases a distancia y los factores psicológicos. Si bien internet es una debilidad durante la docencia, según la respuesta de estos estudiantes, los factores emocionales fueron más relevantes en este período. El análisis de los datos obtenidos de los cuestionarios aplicados mostró que si bien las plataformas adecuadas para la docencia online, así como una buena conexión a internet son fundamentales para que se desarrollen las clases a distancia, los factores emocionales derivados del aislamiento social, la adaptación a las clases y el entorno de estudio son fundamentales fueron los que más obstaculizaron el proceso de aprendizaje de los estudiantes durante el período pandémico.

Palabras clave: Pandemia; COVID-19; Enseñanza online; Aprender en línea; Enseñanza.

\section{Introdução}

O Coronavírus, chamado de SARS-CoV-2 (Síndrome Respiratória Aguda Grave coronavírus 2) e causador da COVID19, é uma infecção respiratória que pode gerar quadros desde assintomáticos até os mais graves, possui alta transmissibilidade e rápida disseminação. Após seu surgimento, a Organização Mundial da Saúde (OMS) decretou a pandemia da COVID-19, estabelecendo recomendações para o controle e contenção do vírus em todo o mundo. Assim, surgiram orientações à população da importância da higienização, principalmente das mãos, e foram determinadas estratégias de distanciamento social.

Por consequências das práticas de distanciamento social adotadas com o intuito de controlar a disseminação do novo coronavírus, no dia 17 de março de 2020, o Ministério da Educação (MEC) aprovou a portaria nº 343 que autorizou a substituição das aulas dos cursos presenciais do ensino superior por aulas remotas, por período em que durar a situação de pandemia da COVID-19. Consequentemente, essa portaria foi alterada pela Portaria no 345 , publicada em 19 de março de 2020 e no dia 16 de junho de 2020 consolidado pela Portaria nº 544 (Brasil, 2020; Brasil, 2020a; Brasil, 2020b).

Portanto, as instituições de ensino superior tiveram suas atividades presenciais suspensas e passaram a realizar suas atividades de forma online. As universidades precisaram mudar a forma de ensinar e se adaptar a essa nova realidade, buscando atender toda a comunidade acadêmica de forma a reduzir os possíveis prejuízos pedagógicos e os riscos à saúde pública e garantir uma educação com qualidade e segurança. As universidades optaram por usar recursos tecnológicos e, com isso, docentes e discentes tiveram que se integrar e aprender novas tecnologias (Garrido \& Garrido, 2020; Bezerra et al., 2020; Gusso et al., 2020; Pontes et al., 2019).

Nesse contexto, algumas universidades optaram pelo ensino remoto emergencial outras pelo ensino a distância (EAD). O ensino remoto é composto por videoconferências com recursos visuais e de áudio em tempo real, com as mesmas disciplinas, professores e horários, continuando assim a interação síncrona entre aluno e professor, só que de forma online, com os planos de ensino e materiais didáticos personalizados por cada docente. Por outro lado, o ensino EAD acontece por meio de vídeo aulas gravadas, possibilitando aos discentes uma maior flexibilização de horário para estudo (Holanda, Pinheiro, \& Pagliuca, 2013; Alonso \& Silva, 2018). A Universidade do Estado de Minas Gerais, durante o período de pandemia, optou pela utilização do ensino remoto emergencial, com as aulas sendo lecionadas por meio de videoconferências e gravadas para os discentes que não conseguiam estar presentes no horário da aula síncrona assistirem em um outro momento. 
O ensino remoto emergencial, não presencial, pode provocar sentimentos de solidão no estudante, deixando-o desmotivado pela necessidade de maior interação, atenção e apoio por partes dos docentes. Emanuelli (2011, p. 210) mostra que essa insatisfação pode ser devido à falta da relação de forma presencial entre professor/estudante e entre colegas de turma. A autora também retrata a dificuldade dos docentes em responder as solicitações individuais da turma, o domínio do uso dos recursos tecnológicos e principalmente as dificuldades com o acesso à internet de maneira síncrona.

Os autores Souza e Reinert (2010, p. 161), apresentaram outro questionamento: a possível ausência do hábito da autoaprendizagem, recaindo sobre a baixa autonomia do estudante, gerando assim, dificuldades em apresentar um papel ativo e interativo. De acordo com Macedo et al. (2018, p. 2), esse papel ativo e interativo traz ao estudante um papel central no processo de ensino e aprendizagem, fazendo com que esta saia de uma relação vertical para uma horizontal, onde o conhecimento não irá se restringir apenas ao professor, mas ao contrário, trazendo um dinamismo ao estudante nas relações de aprendizagem.

Neste contexto, dada a importância da educação superior para a sociedade, este trabalho se objetiva identificar o ambiente que os estudantes do curso de Engenharia da Computação da Universidade do Estado de Minas Gerais, unidade de Divinópolis, estão inseridos, principalmente no que tange, aos recursos tecnológicos os quais possuem para a realização do ensino remoto emergencial, como acesso à internet, qualidade desse acesso, além de verificar através da opinião de estudantes universitário os impactos emocionais gerados pela pandemia em seu processo de aprendizagem.

\section{Metodologia}

Conforme ressaltado por Prodanov e De Freitas (2013) o método científico pode ser definido pelo conjunto de procedimentos realizados que visam garantir o conhecimento em um trabalho científico. Essa definição é pautada nas definições dos termos "método" que consiste em um conjunto de passos para chegar a uma determinada finalidade; e "ciência" que visa a busca pelo conhecimento.

A utilização de procedimentos metodológicos pode ser utilizada não somente pela ciência, no entanto, como destacam Lakatos e Marconi (2007), não se faz ciência sem a utilização de métodos científicos. Desta forma, o presente trabalho utilizase de definições e conceitos acerca da elaboração de uma pesquisa científica definidos por Prodanov e De Freitas (2013), levandose em consideração: natureza, objetivos, procedimentos técnicos, tipo de instrumento que se associa aos procedimentos técnicos e abordagem.

Do ponto de vista de sua natureza, o presente trabalho é pautado pela pesquisa básica, que possui por objetivo produzir conhecimentos que sejam importantes para o avanço de uma área de estudo, mas que, no entanto, não possui aplicação prática em seu desenvolvimento. Para além disso, está relacionada a interesses e verdades a níveis mundiais.

Analisando os objetivos da pesquisa aqui desenvolvida, este trabalho se caracteriza pela pesquisa descritiva, uma vez que os fatos observados na pesquisa são apenas registrados, descritos, analisados, classificados e interpretados sem que estes sofram interferências.

Levando-se em consideração os procedimentos técnicos, este trabalho pode ser subdividido em duas partes, as quais são: pesquisa bibliográfica e levantamento. A pesquisa bibliográfica foi utilizada neste trabalho para se buscar referências na literatura que contribuíssem para sua realização, utilizando-se do instrumento fontes bibliográficas. Já o procedimento técnico denominado levantamento, está associado a pesquisa que envolve a interrogação direta do público o qual se deseja produzir conhecimento, que é exatamente o que propõe o presente trabalho, e como instrumento utilizou-se de formulário on-line, para a coleta de dados da amostra da pesquisa.

Neste contexto, para a realização da coleta de dados primeiramente se fez necessário identificar o público-alvo, que consistiu em estudantes do curso de Engenharia da Computação, da Universidade do Estado de Minas Gerais, unidade de Divinópolis - MG. Ressalta-se que o referido curso está disponível em dois turnos, sendo estes: matutino e noturno, e ambos os 
turnos tiveram dados de estudantes coletados. Salienta-se ainda que foram coletados dados apenas de estudantes que se encontram em períodos ímpares, o que se justifica, uma vez que, as matrículas no referido curso acontecem anualmente.

Como instrumento de coleta de dados, se optou pelo uso da aplicação de formulário on-line, justificável já que a sociedade se encontra em meio a um período pandêmico. $\mathrm{O}$ formulário foi aplicado por 12 dias, compreendidos entre 28 de junho a 09 de julho de 2021, sendo aplicado por meio da plataforma Microsoft Forms. A referida instituição possui uma parceria com a empresa Microsoft, sendo assim todos os estudantes possuem acesso a plataforma, e para este acesso precisam se autenticar, o que garantiu apenas uma resposta por estudante, além de possibilitar que apenas estudantes da instituição tivessem acesso, trazendo maior segurança e confiabilidade.

O formulário se pautou basicamente na identificação de dados relacionados a seis assuntos principais a saber: a) dados relacionados a matrícula do estudante no curso (turno de matrícula e período); b) dados referentes aos dispositivos e tecnologias utilizadas para acesso as aulas (se internet própria, tecnologia da internet, se móvel ou fixa e sobre a qualidade da conexão); c) dados relacionados ao ambiente de estudo; d) dados sobre os hábitos dos estudantes em relação ao uso de plataformas de ensino online; e) sobre como se encontrava emocionalmente para a realização do ensino remoto emergencial; f) e por fim, quais as principais dificuldades encontradas, além de verificar se os estudos foram afetados e qual a influência no aprendizado.

Um ponto importante a se destacar refere-se a determinação da amostra utilizada. Inicialmente, salienta-se que no início do ano letivo de 2021 o curso possuía aproximadamente 330 estudantes matriculados. Sendo assim, para facilitar a coleta dos dados, foi realizado o cálculo amostral, utilizando-se a fórmula de Barbetta, vide (Barbetta (2002)), com significância de 95\% e erro amostral de 5\%. Com a aplicação da referida fórmula, foi verificado que a amostra deveria conter 180 estudantes. Finalmente, o formulário foi enviado para todos os estudantes do curso, havendo assim a possibilidade de todos responderem, sendo o tipo de amostragem definido pela Amostra Aleatória Simples.

Importa salientar que como a coleta de dados utilizada no presente trabalho se pautou apenas em uma pesquisa de opinião, não foi necessária a submissão do projeto para aprovação no Comitê de Ética e Pesquisa, conforme regulamentado pelas resoluções de nº 466/12 CNS (Brasil, 2012) e n 510/16 CEP/CONEP (Brasil, 2016).

Finalmente, do ponto de vista de abordagem do problema foi utilizada a pesquisa quantitativa, uma vez que esta possui por intuito transcrever em números as opiniões e dados coletados por meio dos formulários, para que possam ser classificados e analisados, de modo a gerar conhecimento sobre o assunto estudado. Para que isso fosse possível o formulário se baseou em perguntas cujas respostas pudessem ser quantificadas, não tendo assim nenhuma pergunta de caráter puramente qualitativo.

\section{Resultados e Discussão}

Os questionários foram respondidos por 184 discentes do curso de Engenharia da Computação, sendo 94 (51\%) estudantes do turno noturno e 90 (49\%) do matutino. A internet é considerada um dos fatores mais importantes para que as aulas online aconteçam, diante disso os estudantes foram questionados sobre a forma de acesso à internet, qual a tecnologia usada $\mathrm{e}$ também sobre a estabilidade da conexão. Sendo assim, quando questionados sobre a forma de acesso à internet $183(99,5 \%)$ disseram possuir internet própria e apenas $1(0,5 \%)$ necessita utilizar internet de terceiros, como vizinhos ou estabelecimentos. Além disso, 183 (99,5\%) também disseram utilizar internet fixa e apenas 1 (0,5\%) disse utilizar rede móvel. Já sobre a conexão 138 (75\%) afirmaram que o provedor de internet é estável e que não é comum quedas de sinal ou lentidão e 46 (25\%) disseram que seu provedor de internet sofre constantes oscilações.

Porém, quando questionados com relação ao ambiente, 96 (52,2\%) disseram que é adequado para realizar as atividades online, enquanto 63 (34,2\%) afirmaram possuir ambiente parcialmente adequado e 25 (13,6\%) disseram não possuir (ver Figura 1). É importante mencionar que o ambiente residencial possui uma estrutura diferente da escola e que por mais adequado que seja não foi feito para ser um ambiente educativo, distrações como televisão, contato familiar, dentre outras podem atrapalhar a 
concentração e consequentemente o processo de aprendizagem do estudante. De acordo com os autores dos Santos (2020) e Limeira (2020), o ambiente caseiro permite maior acomodação por parte de alguns estudantes, não favorecendo a atenção e dedicação necessárias aos estudos.

Figura 1 - Quantidade de respostas em porcentagem, relativa à pergunta "O ambiente em que se encontra neste momento de isolamento social é adequado para atividades online?". Pode-se observar que 52,2\% dos alunos possuem ambiente adequado para realizar as atividades remotas.

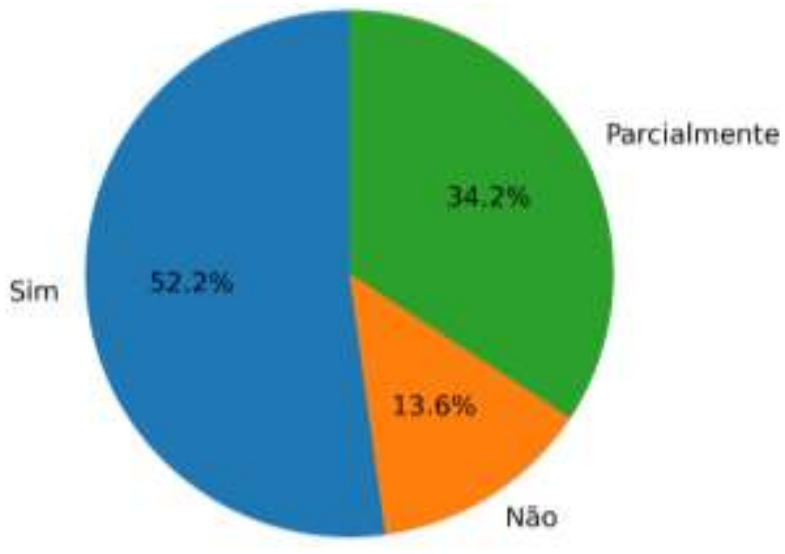

Fonte: Autores.

Apesar do ambiente residencial possuir uma estrutura diferente da encontrada no ambiente escolar, o estudo online por meio de plataformas específicas ou por plataformas gratuitas como Youtube, Podcasts ou similares já era adotado por muitos antes da pandemia do COVID-19 e apesar de 146 (79\%) dos entrevistados terem dito já possuir esse hábito, como pode-se observar na Figura 2, 110 (59,8\%) dos estudantes do curso da Engenharia da Computação que responderam ao formulário pensaram em parar de estudar durante a pandemia (Figura 2). Esse resultado mostra que, provavelmente, fatores além da internet e uso de tecnologias influenciaram na vontade desses alunos em trancar suas matrículas.

Figura 2 - Gráfico das respostas à pergunta “Você pensou em parar de estudar na pandemia?”. Dos 184 entrevistados 110 disseram sim (azul) e 74 não (laranja).



Fonte: Autores.

Vários estudos têm mostrado os impactos da pandemia na saúde mental da população em decorrência da pandemia do novo coronavírus (Rodrigues et al., 2020; Schmidt et al., 2020; Lima, 2020). A situação da quarentena é desagradável devido a diversos fatores, como por exemplo, as incertezas quanto ao futuro, a privação do contato com colegas, amigos e até mesmo 
familiares e pela preocupação com a disseminação da doença. Quando questionados sobre o estado emocional, $52(28,3 \%)$ dos estudantes responderam que se encontram bem, entretanto, 29 (15,7\%) disseram estar incapazes e 103 (56,0\%) parcialmente bem para realizar as atividades online (Figura 3). Além disso, como pode-se observar na Figura 4, 60 (32,6\%), 46 (25,0\%) e 27 $(14,7 \%)$ dos discentes, afirmaram que a pandemia afetou emocionalmente seus estudos parcialmente, muito e extremamente, respectivamente. Mostrando que fatores emocionais podem ser uma das razões que fizeram esses estudantes pensarem em parar de estudar durante a pandemia.

Figura 3 - Quantidade de respostas em porcentagem, relativa à pergunta "Como você se encontra emocionalmente para acompanhar as atividades online?".

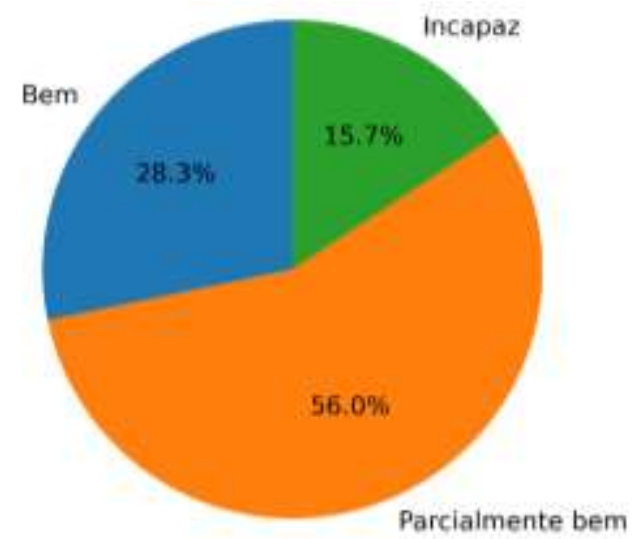

Fonte: Autores.

Figura 4 - Gráfico das respostas à pergunta "Considera que a pandemia afetou emocionalmente seus estudos?".

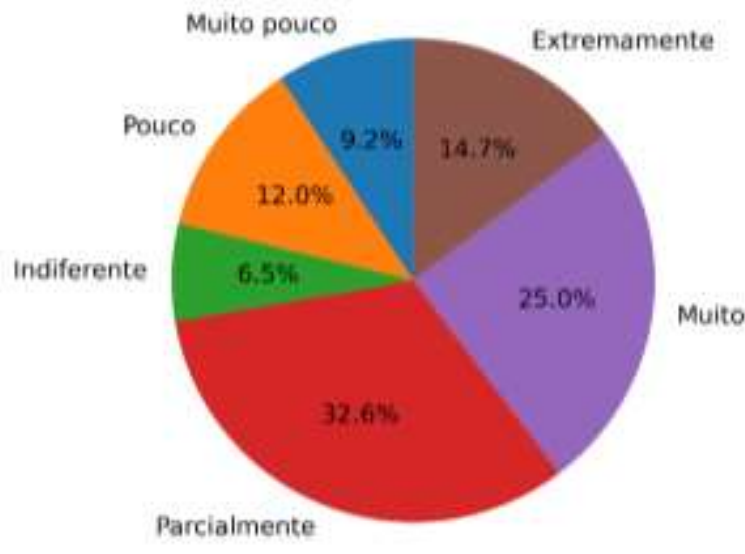

Fonte: Autores.

Porém, além de fatores psicológicos, buscou-se apurar também qual a maior dificuldade encontrada pelos acadêmicos nas aulas remotas. O gráfico da Figura 5 mostra que a adaptação as aulas remotas foi a maior dificuldade encontrada, já que 75 $(40,8 \%)$ dos estudantes relataram este problema, ao passo que 51 (27,7\%) apontaram fatores psicológicos e $27(14,7 \%)$ apontaram o ambiente domiciliar como um dos principais problemas. Cabe salientar que o acesso a internet foi um problema insignificante, se relacionado aos demais. Além disso, 16,3\% relataram não possuir nenhuma dificuldade. A pesquisa demonstrou que não basta ter acesso a internet para que a aula remota aconteça. Inúmeras variáveis interferem no processo educacional fora do ambiente escolar; dentre elas, apontadas neste estudo, o processo de adaptação ao ambiente virtual, as condições inadequadas do ambiente residencial, local onde o aluno fica exposto a toda a situação do entorno familiar, além de fatores psicológicos. 
Figura 5 - A maioria dos alunos do curso da Engenharia da Computação da UEMG-Divinópolis que responderam ao questionário afirma que adaptação as aulas remotas foi a maior dificuldade encontrada durante as aulas remotas. Gráfico com as respostas à pergunta “Qual foi a maior dificuldade encontrada nas aulas remotas?”.

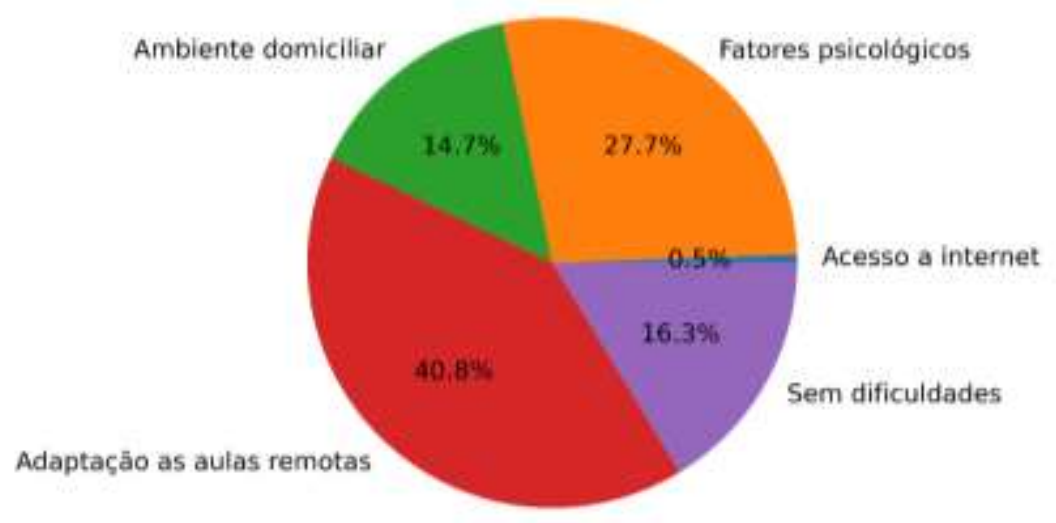

Fonte: Autores.

Por mais que as aulas remotas contribuam de forma significativa no processo de aprendizagem, pois proporcionam ao discente um maior contato com o seu mediador em tempo real para debater e discutir sobre temáticas pré-estabelecidas, propiciando um maior desenvolvimento do acadêmico (Vieira \& Teo, 2018). Pode-se notar por meio da Figura 6 que 85 (46,4\%) e $18(9,8 \%)$ dos entrevistados afirmaram que o ensino remoto influenciou de forma negativa e extremamente negativa, respectivamente, no seu aprendizado. Porém, é interessante mencionar que 40 (21,9\%) destes estudantes disseram que o ensino remoto não influenciou em sua aprendizagem e os outros 40 (21,9\%), divididos entre $14,2 \%$ e $7,7 \%$, julgaram que o ensino remoto influenciou de forma positiva e extremamente positiva em seus estudos.

Figura 6 - Quantidade de respostas em porcentagem, relativa à pergunta "Qual foi a influência da mudança para o ensino remoto em seu aprendizado?".

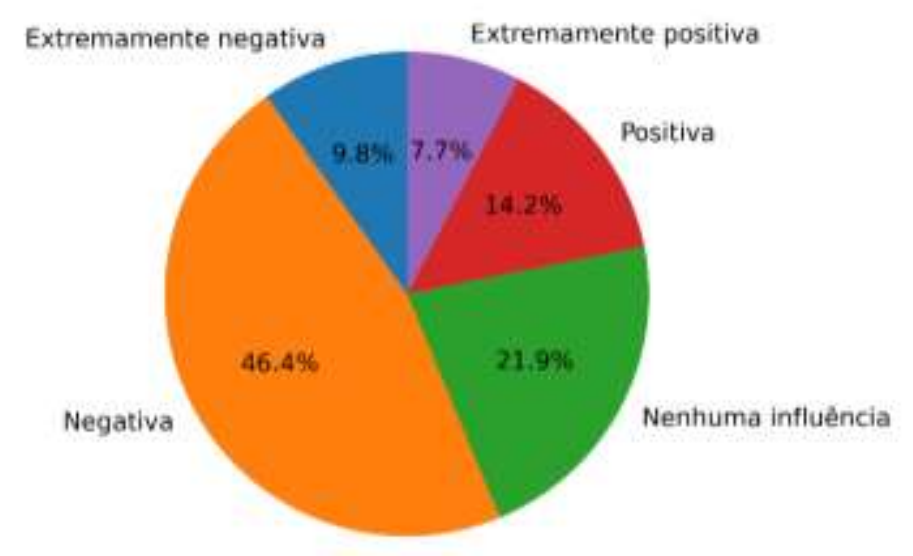

*Um discente não respondeu essa pergunta. Fonte: Autores.

Pelas respostas dos universitários, pode-se perceber que fatores, como internet e acesso a plataformas adequadas apesar de serem de extrema importância para o acompanhamento das aulas, não representaram o mais importante. A presente pesquisa mostrou que fatores emocionais, adaptação as aulas remotas e o ambiente domiciliar foram as maiores dificuldades encontrada pelos alunos do curso da Engenharia da Computação da UEMG. Os estudantes que responderam ao questionário consideraram que o ensino remoto contribuiu de forma negativa para a sua aprendizagem, porém é importante ressaltar que essa experiência 
de aulas remotas aconteceu no meio de uma pandemia, que afetou o emocional da maioria da população brasileira. Um estudo realizado por pesquisadores da Universidade Federal de Santa Maria (UFSM) para acompanhar o impacto da pandemia na saúde mental dos brasileiros, demonstrou que $65 \%$ dos entrevistados tiveram uma piora na saúde mental durante a pandemia (Calegaro, 2020).

\section{Considerações Finais}

Em decorrência da pandemia do coronavírus a vida da sociedade de um modo geral sofreu diversas alterações, uma vez que o distanciamento social passou a ser considerado como um fator importante ao combate da pandemia, principalmente no que tange a disseminação do vírus. Diversas atividades tiveram que ser adaptadas, dentre elas o ensino superior, onde as universidades adotaram o ensino remoto emergencial, como medida paliativa, de modo a minimizar os impactos pedagógicos, além de mitigar os riscos à saúde pública.

Mediante aos pontos que visaram ser investigados neste trabalho, foi possível chegar a algumas conclusões que contribuem para a análise e entendimento do problema abordado. Verificou-se por exemplo, que apesar dos estudantes utilizarem-se de suas próprias residências para a realização das atividades, que $86 \%$ relataram ter ambiente adequado ou parcialmente adequado para as atividades online. Em contrapartida, verificou-se que este não foi um dos pontos mais relevantes aos estudantes neste momento de aulas remotas, já que aproximadamente $60 \%$ relataram ter pensado em parar seus estudos na pandemia. Além disso, pôde-se concluir que aproximadamente 70\% afirmaram que pandemia afetou seus estudos, seja de modo parcial, muito afetado ou extremamente afetado.

Dentre os pontos considerados como maiores limitadores no processo de ensino aprendizado foi possível verificar que a adaptação as aulas remotas, seguido de fatores psicológicos e por fim o ambiente domiciliar foram responsáveis por praticamente $100 \%$ das dificuldades, sendo a adaptação responsável por praticamente $50 \%$ das respostas. Por fim, analisou-se a influência da mudança para o ensino remoto emergencial no aprendizado dos estudantes, onde verificou-se que cerca de $55 \%$ dos estudantes disseram ser ou negativa ou extremamente negativa.

Finalmente, conclui-se que o ensino remoto emergencial, se tratando de uma medida paliativa ao ensino superior, durante a pandemia, possui alguns dificultadores aos estudantes em seu processo de aprendizagem, impactados principalmente pela adaptação do formato, bem como por fatores psicológicos. Entretanto, para uma análise mais efetiva, em trabalhos futuros é necessário uma análise que englobe outros fatores a serem pesquisados, bem como a percepção dos docentes do curso, de modo a contribuir nos resultados identificados.

\section{Agradecimentos}

Agradecemos ao Programa de Apoio a Projetos de Extensão da UEMG - PAEx/UEMG.

\section{Referências}

Alonso, K. M., \& Silva, D. G. D. (2018). A educação a distância e a formação on-line: o cenário das pesquisas, metodologias e tendências. Educação \& Sociedade, 39, 499-514.

Barbetta, P. A. (2008). Estatística aplicada às ciências sociais. Ed. UFSC.

Bezerra, K. P., de Lima Costa, K. F., de Oliveira, L. C., Fernandes, A. C. L., de Carvalho, F. P. B., \& de Sousa Rosso, I. C. A. (2020). Ensino remoto em universidades públicas estaduais: o futuro que se faz presente. Research, Society and Development, 9(9), e359997226-e359997226.

Brasil. (2012). Resolução $\mathrm{n}^{\circ}$ 466, de 12 de dezembro de 2012, do Conselho Nacional de Saúde do Ministério da Saúde. 2012. http://www.conselho.saude.gov.br/resolucoes/2012/Reso466.pdf

Brasil. (2016). Resolução $\mathrm{n}^{\mathrm{o}}$ 510, de 7 de abril de 2016, do Conselho Nacional de Saúde do Ministério da Saúde. 2016. http://conselho.saude.gov.br/resolucoes/2016/Reso510.pdf 
Brasil. (2020). Portaria $n^{\circ} 343$, de 17 de março de 2020. Dispõe sobre a substituição de aulas presenciais por aulas em meios digitais enquanto durar a situação de pandemia do Novo Coronavírus - COVID-19. Ministério da Educação. http://www.planalto.gov.br/ccivil_03/Portaria/PRT/Portaria\%20n\%\%20343-20mec.htm

Brasil. (2020a). Portaria $n^{o}$ 345, de 19 de março de 2020. Altera a Portaria MEC no 343, de 17 de março de 2020. Ministério da Educação. https://pesquisa.in.gov.br/imprensa/jsp/visualiza/index.jsp?jornal=603\&pagina=1\&data=19/03/2020\&totalArquivos=1

Brasil. (2020b). Portaria $n^{\circ} 544$, de 16 de junho de 2020. Dispõe sobre a substituição das aulas presenciais por aulas em meios digitais, enquanto durar a situação de pandemia do novo coronavírus - Covid-19, e revoga as Portarias MEC nº 343, de 17 de março de 2020, n 345, de 19 de março de 2020, e nº 473, de 12 de maio de 2020. Ministério da Educação. https://www.in.gov.br/en/web/dou/-/portaria-n-544-de-16-de-junho-de-2020-261924872

Calegaro, V. C. COVID Psiq: Evolução de sintomas emocionais durante a pandemia. UFSM. https://www.covidpsiq.org/resultados

dos Santos Junior, V. B., \& da Silva Monteiro, J. C. (2020). Educação e covid-19: as tecnologias digitais mediando a aprendizagem em tempos de pandemia. Revista Encantar-Educação, Cultura e Sociedade, 2, 01-15.

Dosea, G. S., do Rosário, R. W. S., Silva, E. A., Firmino, L. R., \& dos Santos Oliveira, A. M. (2020). Métodos ativos de aprendizagem no ensino online: a opinião de universitários durante a pandemia de COVID-19. Interfaces Científicas-Educação, 10(1), 137-148.

Emanuelli, G. B. (2011). Atração e refração na educação a distância: constatações sobre o isolacionismo e a evasão do aluno. Revista Gestão Universitária na América Latina-GUAL, 4(2), 205-218.

Garrido, R. G., \& Garrido, F. D. S. R. G. (2020). COVID-19: um panorama com ênfase em medidas restritivas de contato interpessoal. Interfaces CientíficasSaúde e Ambiente, 8(2), 127-141.

Gusso, H. L., Archer, A. B., Luiz, F. B., Sahão, F. T., Luca, G. G. D., Henklain, M. H. O., \& Gonçalves, V. M. (2020). Ensino superior em tempos de pandemia: diretrizes à gestão universitária. Educação \& Sociedade, 41.

Holanda, V. R. D., Pinheiro, A. K. B., \& Pagliuca, L. M. F. (2013). Aprendizagem na educação online: análise de conceito. Revista Brasileira de Enfermagem, 66, 406-411.

Lima, R. C. (2020). Distanciamento e isolamento sociais pela Covid-19 no Brasil: impactos na saúde mental. Physis: Revista de Saúde Coletiva, 30.

Limeira, G. N., Batista, M. E. P., \& de Souza Bezerra, J. (2020). Desafios da utilização das novas tecnologias no ensino superior frente à pandemia da COVID19. Research, Society and Development, 9(10), e2219108415-e2219108415.

Marconi, M. A., \& Lakatos, E. M. (2007). Metodologia científica. Atlas.

Macedo, K. D. D. S., Acosta, B. S., Silva, E. B. D., Souza, N. S. D., Beck, C. L. C., \& Silva, K. K. D. D. (2018). Metodologias ativas de aprendizagem: caminhos possíveis para inovação no ensino em saúde. Escola Anna Nery, 22.

Pontes, A. P. F. F., da Silva, N. R., \& Barboza, P. L. (2019). Professor de matemática e a utilização das tecnologias no ensino: realidade x expectativa. Research, Society and Development, 8(3), 01-18.

Prodanov, C. C., \& De Freitas, E.C. (2013). Metodologia do trabalho científico: métodos e técnicas da pesquisa e do trabalho acadêmico-2a Edição. Editora Feevale.

Rodrigues, B. B., Cardoso, R. R. D. J., Peres, C. H. R., \& Marques, F. F. (2020). Aprendendo com o Imprevisível: Saúde mental dos universitários e Educação Médica na pandemia de Covid-19. Revista Brasileira de Educação Médica, 44.

Schmidt, B., Crepaldi, M. A., Bolze, S. D. A., Neiva-Silva, L., \& Demenech, L. M. (2020). Saúde mental e intervenções psicológicas diante da pandemia do novo coronavírus (COVID-19). Estudos de Psicologia (Campinas), 37.

Souza, S. A. D., \& Reinert, J. N. (2010). Avaliação de um curso de ensino superior através da satisfação/insatisfação discente. Avaliação: Revista da Avaliação da Educação Superior (Campinas), 15, 159-176.

Vieira, V. B. R., \& Teo, C. R. P. A. (2018). O ensino a distância na formação em saúde. Revista de Educação Popular, 17(1), 114-125. 